\title{
With high sensitivity and with wide-dynamic-range Localized surface-plasmon resonance sensor for volatile organic compounds
}

\author{
Akira Monkawa ${ }^{\text {a, } * \text {, Tomoe Nakagawa }}{ }^{a}$, Hirokazu Sugimori ${ }^{a}$, Elito Kazawa ${ }^{a}$, \\ Kohei Sibamoto $^{\mathrm{b}}$, Takashi Takei ${ }^{\mathrm{b}}$, Masatake Haruta ${ }^{\mathrm{b}}$ \\ ${ }^{a}$ Tokyo Metropolitan Industrial Technology Research Institute, 2-4-10, Aomi, Kotoku, \\ Tokyo 135-0064, Japan \\ ${ }^{b}$ Graduate School of Urban Environmental Sciences, Tokyo Metropolitan University, \\ 1-1, Minami-osawa, Hachioji-shi, Tokyo 192-0397, Japan \\ E-mail: monkawa.akira@iri-tokyo.jp
}

Fax: +81-3-5530-2629 


\section{Abstract}

To detect volatile organic compounds (VOCs), optical sensors based on the localized surface-plasmon resonance (LSPR) are widely used because they are simple and inexpensive to fabricate. Although LSPR spectroscopy has been generally applied to biological molecules such as proteins and DNA, small molecular sensing has been difficult because of the very small changes induced in the refractive index. We describe a LSPR gas sensor with high and wide range sensitivity. These characteristics are obtained from the improved peak shift that results from using an Au-dot pattern coated with a porous silica film. The 400-nm-diameter, 800-nm-wide Au dots were patterned by electron beam lithography (EBL) on a quartz glass substrate. The LSPR peak of the isolated Au-dot pattern occurs at $1230 \mathrm{~nm}$ in the near isolated area bringing enough refractive index changes. In addition, coating the Au-dot pattern with a thin porous silica film increases the adsorption of VOCs, thereby increasing the refractive-index change. The response of the sensor to VOC vapors was measured in a dynamic-flow system. The relative intensity decreases with toluene vapor exposure but completely recovers to the initial baseline after exposure to clean air. The real-time response allows detection of toluene vapors with concentrations ranging from 1 to $26000 \mathrm{ppm}$, and the limit of detection for toluene is calculated to be $0.4 \mathrm{ppm}$. Eighteen VOC vapors were 
tested and each type exhibited different calibration curves, but these differences depend on the adsorption characteristics of porous silica rather than on the refractive index of the VOCs.

Keywords:

Volatile organic compounds

Localized surface-plasmon resonance

Gold dot pattern

Porous silica film 


\section{Introduction}

Volatile organic compounds (VOCs) are emitted as gases from certain solids or liquids and may contain a variety of chemicals with adverse short- and long-term health effects [1]. To reduce the amount of suspended particulate matter and photochemical oxidants in air, the United States and other nations have developed new regulations to control VOC emissions from factories [2]. VOCs are also present in some workplaces, especially in the chemical industries such as coating and drying facilities, industrial cleansing and drying facilities, printing and drying facilities, VOCs storage facilities (e.g., gasoline, etc.), and facilities that use adhesives. Methods to remove VOCs have been rapidly introduced into these facilities to safeguard worker health and also to reduce atmospheric emissions to avoid environmental hazards [3]. Physical, chemical, and biological treatments are utilized to remove VOCs from air by either recovery or destruction. To operate these systems, the concentration of the vapors must be monitored.

Various VOC-monitoring methods have been developed, such as flame ionization detection (FID), photoionization detection (PID), and nondispersive infrared (NDIR) detection [4]. These are standard methods to detect VOCs and each method has its own advantages and disadvantages. FID is commonly used for VOC monitoring and gives 
direct reading of the total organic carbon (TOC) content. The FID method is based on detecting ions formed during pyrolysis of VOCs in a hydrogen flame. FID cannot be used where there is a potential risk of explosion because a hydrogen flame is used to ionize the solvent. The PID method is similar to FID, except that the solvent is ionized by using a high-voltage ultraviolet (UV) lamp and no hydrogen is required. The NDIR method is based on detecting the amount of IR energy absorbed by the VOCs. NDIR monitors require no fuel, unlike FIDs. PID and NDIR devices are sensitive but expensive and lack specificity. The PID UV lamps can fail and are affected by high humidity.

Because they are compact and inexpensive, VOC sensors serve as leak detectors in VOC removal systems. The VOC sensor performance is determined by its sensitivity, selectivity, simplicity, rapidity, and reliability. Several other VOC-sensing techniques exist such as using semiconductor-metal oxides (e.g., $\mathrm{SnO}_{2}, \mathrm{TiO}_{2}, \mathrm{ZnO}$ ) [5-7], surface acoustic waves (SAWs) [8, 9], and quartz crystal microbalances (QCMs) [10]. Each sensing technique has its own inherent advantages and limitations, which determines in which field each sensor is applied and what service is performed.

Because it is simple and inexpensive to fabricate them, optical sensors for detecting VOCs may be applied across a wide range of fields. The surface plasmon resonance 
(SPR) phenomenon is a very sensitive optical phenomenon and so is used in chemical sensing [11-17]. When a surface plasmon forms on an Au-dot nanopattern, the SPR energy is confined to the isolated dots. It is called localized surface plasmon resonance (LSPR). An LSPR is a collective electron-density oscillation concentrated locally around elements of a metallic nanopattern and is excited by electromagnetic radiation. The metallic nanopattern exhibits a strong resonance whose characteristics depend on the material, the geometry of the nanopattern, and the surrounding medium $[18,19]$. The presence of organic molecules such as protein and DNA shifts the LSPR resonance peak, which allows the molecules to be detected [20, 21]. Organic molecules have a higher refractive index than clean air. Therefore, when they bind to the dot surfaces the local refractive index increases, shifting the resonance peak to a higher wavelength.

We report herein the characteristics of LSPR spectra from Au and Ag nanoparticles and from $\mathrm{Au}$ nanoshells [22]. In the proposed LSPR sensor, metallic nanopatterned substrates are functionalized with various thiolate self-assembled monolayers and tetraoctylammonium bromide is applied to improve selectivity to various VOCs $[23,24]$. By using $\mathrm{Ag}$ and $\mathrm{Au}$ nanosphere films, a high-resolution LSPR-based sensor is demonstrated by detecting extremely low concentrations of inert gas (Ar, $\mathrm{N}_{2}$, and $\mathrm{He}$ ) $[25,27]$. In addition, sensors based on Ag nanoparticles in a metal-organic framework 
material enhance the signal for $\mathrm{CO}_{2}$ sensing.

To systematically examine the LSPR, recent studies proposed methods to fabricate metal nanopatterns with elements of homogeneous size and shape. Simple methods to fabricate metal nanopatterns include natural lithographies such as nanosphere lithography and colloidal lithography [28-29]. These methods are massively parallel and offer an inexpensive and rapid method to fabricate a large array of nanostructured materials. However, because the diameter and pitch of the dots are difficult to control, the size heterogeneity and aggregation of particles prevent the formation of a uniform nanopattern. Direct-write methods, such as electron beam lithography (EBL) [30], focused-ion-beam (FIB) milling [31], and dip-pen lithography [32], provide ideal metal nanopatterns with well-controlled size, shape, and lattice spacing, as well as few defects. Although these methods do present some disadvantages such as the serial nature of the process and higher cost, they are the most suitable for fabricating patterns of complicated shape.

We now report a high-sensitivity wide-range LSPR sensor. The adsorption of VOCs on the sensor surfaces shifts the resonance peak. VOC molecules are adsorbed upon the surface with increasing in VOC concentration. Because VOCs have a higher refractive index than clean air, the local refractive index increases with their adsorption, causing a 
redshift in the LSPR spectrum. For high-sensitivity detection of VOCs, it is necessary for VOC adsorption to produce a large shift in the LSPR spectrum. The desired sensitivity is obtained by two contrivances; coating the Au-dot pattern with a thin porous silica film and positioning the initial LSPR peak in near infrared. Porous silica materials such as mesoporous silica and silica gel are known to be good adsorbers of VOC gases [33]. The porous silica film increases the sensitivity to VOCs by enhancing VOC adsorption, thereby increasing the change in refractive index. The position of the LSPR peak is also critical to maximize the sensitivity. The shift of LSPR peak with VOC adsorption depends on the characteristics of the Au nanopattern [23]. In this study, we used a finite-difference time-domain (FDTD) simulation to design a nanopattern that gives maximum sensitivity to VOCs.

\section{Experimental}

\subsection{Fabrication of Au nanopattern.}

The Au nanopattern was fabricated on a quartz glass substrate by EBL. The substrate was first immersed in cleaning solution $\left(70 \% \mathrm{H}_{2} \mathrm{SO}_{4}\right.$ and $\left.30 \% \mathrm{H}_{2} \mathrm{O}_{2}\right)$, sonicated for 30 min, and then rinsed with deionized water. A thin Cr layer $(\sim 1 \mathrm{~nm})$ was deposited on the substrate to make it conductive. A $350 \mathrm{~nm}$ layer of ZEP520A photoresist mixed in a 1:1 
ratio with the solvent Anisol was spin coated onto the Cr-coated substrate, and then the substrate was baked for $3 \mathrm{~min}$ at $180^{\circ} \mathrm{C}$. The resist layer was then patterned by exposure to an electron beam using an Erionix EBL 7500EX. After exposure, the substrate was immersed in chromium etchant solution 1020 for 10 to $15 \mathrm{~s}$ and then rinsed with deionized water. The substrate was then developed in ZEDN50, rinsed in methyl isobutyl ketone diluted 1:3 in isopropyl alcohol, dipped in isopropyl alcohol, and gently dried. The Cr layer was removed by Ar-ion etching. We used the spatter method to deposit a 40-nm-thick $\mathrm{Au}$ layer on the patterned substrate. Finally, the residual photoresist pattern was removed by sonicating the substrate in dimethylacetamide for 15 min. Images of the nanopattern on glass were acquired with a scanning electron microscope (SEM). The LSPR spectra were acquired by UV-visible near-IR (NIR) spectroscopy (Shimadzu SolidSpec-3700 double-beam system with monochromator).

\subsection{Porous silica coating on Au nanopattern.}

A porous silica thin film was deposited on the Au nanopattern by spin coating with cetyltrimethylammonium bromide (CTAB) as surfactant. Initially, CTAB was dissolved in distilled water at $60^{\circ} \mathrm{C}$. The CTAB solution was adjusted to $\mathrm{pH} 2.0$ by adding $\mathrm{HCl}$. Next, tetraethyl orthosilicate (TEOS) was slowly added and the solution was stirred for

$3 \mathrm{~h}$. The final precursor composition was $1 \mathrm{CTAB}, 8 \mathrm{TEOS}$, and $185 \mathrm{H}_{2} \mathrm{O}$. The solution 
was dropped onto the gold nanopattern substrate, which was spun at up to $5000 \mathrm{rpm}$ for $1 \mathrm{~min}$. The substrate was then placed in an oven at $100^{\circ} \mathrm{C}$ for $1 \mathrm{~h}$. Next, the template was removed by calcination at $450^{\circ} \mathrm{C}$ for $1 \mathrm{~h}$ with a heating and cooling ramp of $20^{\circ} \mathrm{C}$ /min. The thickness of the porous silica thin film on the gold nanopattern was measured by using a field-emission scanning electron microscope (S-4800 FE-SEM: Hitachi High-Technologies, Japan). Nitrogen adsorption-desorption of calcined porous silica film was measured at $77 \mathrm{~K}$ with a BellSorp-max (Bell Japan Inc.). The porous silica film was deposited at submicron thickness on glass. The film was degassed under vacuum at $150^{\circ} \mathrm{C}$ for $3 \mathrm{~h}$ before measuring adsorption-desorption. We used the Brunauer-Emmet-Teller (BET) analysis and the Dollimore-Heal (DH) method to calculate the specific surface area and pore-size distribution, respectively.

\subsection{Vapor-generation system.}

To evaluate the performance of the LSPR sensor, we fabricated a vapor-generation system to provide variable organic vapor concentrations. Fig. 1 shows the schematic diagram of VOC sensing system. Dry air (humidity $<1 \%$ ) generated by clean air unit (P4-QD10, IAC) was used as base gas in the sensing system. The system used a bubbling method and a diffusion method. The bubbling method generated variable organic vapor concentrations at room temperature by diluting saturated VOC gases with 
dry air. The concentration range of toluene gas ranged from 100 to $26000 \mathrm{ppm}$. Low-concentration VOC gases (1-100 ppm) were obtained by using a standard gas-generation system (Permeater PD-1B-2, GASTEC) that is based on the diffusion method. The diffusion method generates low-concentration VOC gases because the gas concentration is determined from the diffusion rate of evaporated VOCs in a small tube. The flow rate of VOC gases was controlled by a mass-flow controller (MODEL8500, KOFLOC). The vapor concentrations were calibrated with a gas chromatograph FID (GC-2010, Shimadzu).

\subsection{VOC sensing system.}

The VOC sensing system was used as a reflection LSPR-based optical fiber sensor. The sensor consisted of a light source, a chamber, and a spectrometer. The chamber was connected to the spectrometer and the light source through $100 \mu \mathrm{m}$ optical fibers. The light source was a tungsten halogen lamp (LS-1, Ocean Optics) optimized for the visible-NIR (VIS-NIR) range (360-2500 nm). LSPR spectra were measured using a real-time CCD-array UV-VIS-NIR spectrometer (NIRQuest, Ocean Optics). The LSPR sensor chip was placed in the chamber for exposure to VOC gasses.

\subsection{The characterization of the LSPR sensor chip.}

Three parameters of sensitivity, response and recovery time were used to characterize 
sensor performance. Sensitivity is a change of measured signal per VOC gas concentration unit. Response is the time required for sensor to respond to a step concentration change from zero to a certain concentration value. Recovery time is the time it takes for the sensor signal to return to its initial value after a step concentration change from a certain value to zero.

\section{Results and Discussion}

\subsection{Design of LSPR sensor.}

Fig. 2 shows the LSPR spectra calculated by OptiFDTD for five different Au-dot patterns [34]. Gold dots had diameters ranging from 100 to $500 \mathrm{~nm}$ and pitches from 200 to $1000 \mathrm{~nm}$. The calculated LSPR peaks of D100P200, D200P400, D300P600, D400P800, and D500P1000 appear at 600, 750, 1000, 1270, and $1550 \mathrm{~nm}$, respectively.

The LSPR spectra were determined based on two parameters: diameter and pitch.

Based on the results of the FDTD simulation, we prepared five different types of Au-dot patterns. Fig. 3 shows SEM images of Au-dot patterns fabricated by EBL. The LSPR spectra (Fig. 4) of the Au-dot patterns show that the LSPR peak position shifts to higher wavelengths as the diameter and pitch of the Au dots increase. The LSPR peaks of D100P200, D200P400, D300P600, D400P800, and D500P1000 appear at 750, 850, 
1000, 1250, and $1550 \mathrm{~nm}$, respectively. LSPR peaks of D100P200 and D200P400 differ from those estimated by calculation because the error in dot size increases as the diameter and pitch become smaller.

To enhance the sensitivity of the LSPR sensor, it is important to understand, for each peak wavelength, how the LSPR peak shifts as a function of changes in refractive index of the Au-dot-patterned surface. A previous study [35] reported that this shift is greater in the NIR than that in the visible. We estimated the LSPR peak shift for five Au-dot patterns by using an ethanol-solution drop test. The shift for D400P800 was similar to that of D500P1000, and these were the largest shifts for all the Au-dot patterns. This result indicates that the LSPR spectrum in the NIR is appropriate for obtaining the desired sensor sensitivity.

In the NIR, the LSPR spectrum is strongly influenced by water vapor in the atmosphere because water absorption bands occur at 1400 and $1900 \mathrm{~nm}$. Thus, to avoid deformation of the LSPR peak profile by moisture adsorbed on the sensor surface, the LSPR peak position should be adjusted to occur below $1300 \mathrm{~nm}$. Because the spectrum of the Au-dot pattern of D 400 P 800 satisfies this condition, we chose D 400 P 800 as Au-dot pattern for the proposed LSPR sensor.

The sensitivity of the LSPR sensor chip was confirmed by using toluene gas at 
concentrations ranging from 100 to $1000 \mathrm{ppm}$. For all gas concentrations, the LSPR peak position remains the same before and after induction of the gas. The LSPR chip of bare Au-dot pattern has insufficient sensitivity. The LSPR sensor is generally sensitive to changes in the refractive index of substances adsorbed on the dot-patterned surfaces. Low concentrations of VOC gas, i.e., gas from organic compounds of low volatility, can be detected by retaining gas molecules at the sensor surfaces at the concentration needed to produce the required change in refractive index. The porous silica thin film serves to this purpose: its microstructure consists of many nanopores, which can lead to a condensed layer forming on the sensor surfaces by capillary condensation. The condensed organic layers caused the surface refractive index to change from air to a portion of the organic liquid. Note that the porous silica coating does not alter the profile of the LSPR spectrum because its extinction coefficient is essentially zero. These characteristics are indispensable to the enhanced performance of the sensor.

The porous silica thin film was fabricated by spin coating from a precursor solution, resulting in 300-nm-thick coatings as measured by FE-SEM. The specific surface area was $797.9 \mathrm{~m}^{2} / \mathrm{g}$, the pore volume was $0.53 \mathrm{~cm}^{3} / \mathrm{g}$, and the average pore size was 2.67 nm. A previous study [36] reported that the amount of toluene adsorbed on $\mathrm{SiO}_{2}$ powder increased with increasing specific surface area but tended to saturate at about $900 \mathrm{~m}^{2} / \mathrm{g}$ 
because of the relationship between $\mathrm{SiO}_{2}$-pore size and the size of adsorbent molecules.

The specific surface area found in the present study is similar to that found in the previous study [36]; thus, we concluded that porous silica thin film was the most suitable film for VOC adsorption.

Fig. 5 shows the change of the LSPR spectrum as a result of coating with porous silica. The peak maximum shifts from 1260 to $1232 \mathrm{~nm}$ and the peak width decreases to approximately one-third of its original value. In addition, a new peak appears at $930 \mathrm{~nm}$. These changes are due to annealing, which occurs during the fabrication of the porous silica. A previous study [37] reported that the LSPR peak from Ag nanoparticle arrays shifts to lower wavelengths and the peak width decreases during annealing at $300^{\circ} \mathrm{C}$. These results are supported by atomic-force microscopy observations that $\mathrm{Ag}$ nanoparticles change from triangular to ellipsoidal upon annealing. In contrast, annealing also causes oxidation of the thin Cr layer. According to the FDTD simulation, the localized electromagnetic field is weakened by the high extinction coefficient of the thin $\mathrm{Cr}$ layer. As a result, the LSPR peak is considerably broader with the thin Cr layer than without it. Upon annealing, the thin $\mathrm{Cr}$ layer transforms into $\mathrm{Cr}_{2} \mathrm{O}_{3}$. The localized electromagnetic field after coating with porous silica is similar to that without the thin $\mathrm{Cr}$ layer because the extinction coefficient of $\mathrm{Cr}_{2} \mathrm{O}_{3}$ is negligibly small. This conclusion 
is supported by the appearance of a new LSPR peak at $920 \mathrm{~nm}$ (Fig. 3). The peak also appears in the simulated LSPR spectrum for a Cr-free device, which suggests that the presence of $\mathrm{Cr}$ causes the peak at $920 \mathrm{~nm}$ to disappear.

\subsection{VOC sensing by LSPR sensor.}

Fig. 5 shows the changes in the LSPR spectrum upon exposure to gases with different relative toluene concentrations (0-100 vol\%). With increasing toluene concentrations, the LSPR spectrum shifts to higher wavelength and the peak maximum slightly decreases. Because the refractive index of toluene is higher than that of air, this change to adsorption of toluene onto the porous silica. We observed the dynamic response of the LSPR sensor at $1270 \mathrm{~nm}$, which is the maximum difference in intensity at each wavelength estimated from the difference in spectra between 0 vol\% and 100 vol\% toluene concentration.

Fig. 7 shows the dynamic response of the LSPR sensor for toluene gas concentrations ranging from 10 to $20000 \mathrm{ppm}$. To confirm the relative-intensity recovery ratio, the sensor was exposed to decreasing concentrations of toluene vapors, starting at 20000 ppm, with two measurements taken at each concentration. From 20000 to 2700 ppm, the sensor was exposed to a given concentrations for $100 \mathrm{~s}$, and then exposed to clean air for $900 \mathrm{~s}$. From 1000 to $10 \mathrm{ppm}$, the respective exposure times were $100 \mathrm{~s}$ and $600 \mathrm{~s}$. 
When the toluene gas was introduced, the LSPR peak red shift was observed as decreased in relative intensity. The spectrum returns to its original profile upon purging with clean air, which means that desorption of toluene from the porous silica is complete. However, the recovery time becomes longer when toluene concentration was increased. The spectral profile is similar to that expected for gas adsorption on the porous material [38]. Repetitive tests indicate that the sensor response is highly repeatable at each concentration. Spectra from five repeated pulses of toluene gas at $20000 \mathrm{ppm}$ and $100 \mathrm{ppm}$ were acquired to determine reproducibility. The relative standard deviations of $20000 \mathrm{ppm}$ and $100 \mathrm{ppm}$ were less than $1 \%$, indicating that the results from repeated use of the sensor are very consistent.

Fig. 8a shows toluene adsorption isotherms estimated by using the LSPR sensor, and Fig. $8 \mathrm{~b}$ shows toluene adsorption isotherms from static adsorption experiments with porous silica powder. The results of both measurements are very similar and agree with the Langmuir model, which models chemical adsorption in micropores such as in activated carbon and zeolite [39, 40]. The adsorption isotherm for the bare Au-dot pattern $^{19}$ is linear and differs from that of porous silica. Therefore, the sensor detects toluene molecules adsorbed in the porous silica layer.

\subsection{High sensitivity of sensor.}


Fig. 9 shows the dynamic response of the sensing element in the presence of toluene at concentrations ranging from 1 to $50 \mathrm{ppm}$. The sensor was exposed to given toluene concentrations for $300 \mathrm{~s}$, then switched off for $600 \mathrm{~s}$. The response and recovery times are in the range of a few seconds and the recovery is almost complete. For a toluene concentration of $1 \mathrm{ppm}$, the change is $30-35$ counts, which is much larger than the standard deviation $\sigma$ in baseline intensity of 1.42 . Fig. 10 shows the calibration curves obtained from the dynamic response taken every $50 \mathrm{~s}$ after the introduction of toluene gas. The straight line is a fit to the data acquired at $600 \mathrm{~s}$ and has a slope $s$ of 10.93 $\mathrm{ppm}^{-1}$. The response of the sensor is approximately linear in concentration over the entire investigated range. The limit of detection (LOD) estimated from $3 \sigma / s$ is $0.4 \mathrm{ppm}$ for toluene. Spectra from five repeated pulses of toluene gas at $1 \mathrm{ppm}$ were acquired to determine reproducibility. The relative standard deviation was about $25 \%$.

\subsection{Selectivity of LSPR sensor.}

Fig. 11 shows the calibration curves for (a) aromatic, (b) alcohol, (c) chloroethylene, and (d) chloromethane VOCs in the range of 10 to $1000 \mathrm{ppm}$. The slope of the calibration curves, LOD, and vapor pressure of the tested VOCs are listed in Table 1. Fig. 12 shows that intensity increases with concentration of VOC and that the profile of the calibration curves differ for each VOC. Aromatic, chloroethylene, and 
chloromethane VOCs generally have linear calibration curves. Alcohol VOCs have nonlinear calibration curves with an inflection point near $100 \mathrm{ppm}$.

Among the seven vapors of aromatic VOCs tested, o-xylene, m-xylene, p-xylene, and ethylbenzene have similar and fairly linear responses. This result is supported by their similar slopes for concentration ranges $10-100 \mathrm{ppm}$ and 100-1000 ppm. Toluene has a nonlinear calibration curve with an inflection point near $100 \mathrm{ppm}$, corresponding to the change in slope between the upper and lower concentration ranges, i.e., 10-100 ppm and 100-1000 ppm, respectively. Although the calibration curve of chlorobenzene is similar to that of toluene, it is more linear due to similar slopes between the upper and lower concentration ranges. The five alcohol VOC vapors tested exhibit different calibration curves. The slopes for the chloroethylene and chloromethane VOC groups are small and similar to those for benzene and methanol. These VOCs are characterized by high saturated vapor pressures. LODs for these VOCs are estimated based on the slopes at 10-100 ppm (Table 1). For aromatic and alcohol VOCs, LODs are less than 1 ppm, except for benzene and methanol. For chloroethylene and chloromethane VOCs, LODs are higher than those of aromatic and alcohol VOCs. These results are attributed to the high saturated vapor pressure of the chloroethylene and chloromethane VOC groups. The LOD of m-xylene is calculated to be $0.32 \mathrm{ppm}$, which is considerably lower 
than found in previous studies [23].

Fig. 13 shows adsorption isotherms of (a) aromatic, (b) alcohol, (c) chloroethylene, and (d) chloromethane VOCs. Aromatic VOCs saturate after a linear increase in intensity up to $P / P_{0} \sim 0.2$ to 0.3 . These isotherms are associated with ideal type-I isotherms in the IUPAC classification [41] of adsorption isotherms for gas-solid equilibria. The adsorption process is usually complete at a partial pressure of $\sim 0.5$. A type-I isotherm is typical of adsorption onto a predominantly microporous structure, which is consistent with aromatic VOCs adsorbed in micropores of the thin layer of porous silica. The adsorption characteristics reveal strong adsorbate-adsorbent interactions. Alcohol VOCs have two inflection points: $P / P_{0}=0.2$ and $P / P_{0}=0.35$. The methanol isotherm is associated with a type-II isotherm, which corresponds to unrestricted monolayer-multilayer adsorption on a nonporous solid. The adsorption results thus indicate that methanol molecules form a multilayer after completion of the monolayer without adsorption into micro and mesopores in the thin layer of porous silica. Other alcohol VOC isotherms are similar to a type-VI isotherm, which is a variation of type II but includes the formation of a finite multilayer that corresponds to complete filling of the capillaries within the mesopore structure. The difference with type II is monolayer adsorption on the mesopore walls. The adsorption process indicates 
that other alcohol VOCs undergo monolayer-multilayer adsorption on the mesopore walls. For chloroethylene and chloromethane VOCs, the intensity is small at low pressure compared with aromatic and alcohol VOCs. The isotherms for dichloromethane are similar to a type-II isotherm. Other VOCs isotherms have characteristics intermediate between type-IV and type- $\mathrm{V}$ isotherms. Type- $\mathrm{V}$ isotherms are characteristic of weak adsorbate-adsorbent interactions. The adsorption process indicates that chloroethylene and chloromethane VOCs are weakly adsorbed in porous silica.

The shift in wavelength of the LSPR peak is related to the refractive index of the adsorbate on the sensor surfaces. In adsorption tests of VOCs at a liquid-solid interface, results from a porous-silica-coated LSPR sensor indicate a clear interaction between the magnitude of the wavelength shift in the LSPR peak and the VOC refractive index. However, in VOC gases, there is no correlation between the two, as shown in Fig. 13. The porous silica was completely covered in VOC solution, but VOC gas and air were still present in the porous silica. The ratio of VOC gas and air depends on the adsorption parameters such as structural porosity and the saturated vapor pressure of the gas. Fig. 14 shows the interaction between sensor sensitivity (count/ppm) and the reciprocal of VOC saturated vapor pressure $(1 / \mathrm{Pv})$. The results show that the sensitivity increases 
linearly with decreasing pressure, which indicates that the sensitivity depends on VOC vapor pressure. Therefore, the evidence strongly suggests that the shift of the LSPR peak is mainly affected by the adsorption properties rather than by the refractive index of the VOCs.

\section{Conclusions}

We have fabricated a highly sensitive and wide-range LSPR sensor based on an Au-dot pattern. High sensitivity was obtained by coating the Au-dot pattern with porous silica. These LSPR sensors are very sensitive to the refractive index of the substances adsorbed on the Au-dot-patterned surfaces. Furthermore, the sensitivity increases with the thickness of the surface condensation layer. To improve the sensitivity of the LSPR sensor, the large number of VOC molecules must be adsorbed on the surface. The porous structure of thin porous silica film fabricate VOC gas to condense into liquid, and these condensed layers cause the surface refractive index to change from that of air to that of the organic liquid. By increasing the adsorption capacity, the porous silica coating on the surface of Au dots leads to a sufficiently large change in refractive index. The dynamic response of the LSPR sensor to changes in vapor concentration is rapid, reversible, and reproducible. Eighteen VOC vapors were tested and each type exhibited different calibration curves, but these differences depend on the adsorption 
characteristics of porous silica for VOCs rather than on the refractive index of the VOCs.

\section{Acknowledgement}

This research was supported in part by the grant programs, "The Local Collective Research Development Program” in the Japan Science and Technology Agency (JST) and "Technological Strategies for Solving Urban Issues Program" Bureau of Industrial and Labor Affairs, Tokyo Metropolitan Government.

\section{References}

[1] M. Kampa, E. Castanas, Human health effects of air pollution, Environtal Pollution $151(2008) 362-367$.

[2] F.I. Khan, A.K. Ghoshal, Removal of Volatile Organic Compounds from Polluted Air, Journal of Loss Prevention in the Process Industries 13 (2000) 527-545.

[3] A. Berenjian, N. Chan, H. Malmiri, Volatile Organic Compounds Removal Methods: A Review, American Journal of Biochemistry and Biotechnology 8 (2012) $220-229$. 
[4] B. Yuan, M. Shao, J. de Gouw, D.D. Parrish, S. Lu, M. Wang, L. Zeng, Q. Zhang, Y. Song, J. Zhang, M. Hu, Volatile organic compounds (VOCs) in urban air: How chemistry affects the interpretation of positive matrix factorization (PMF) analysis Journal of Geophysical Research: Atmospheres 117 (2012) D24.

[5] W. Zeng, T.M. Liu, Z.C. Wang, Enhanced Gas Sensing Properties by $\mathrm{SnO}_{2}$ Nanosphere Functionalized. $\mathrm{TiO}_{2}$ Nanobelts, Journal of Materials Chemistry 22 (2012) 3544-3548.

[6] M.H. Seo, M. Yuas, T. Kida, J. S. Huh, N. Yamazoe, K. Shimanoe, Microstructure control of $\mathrm{TiO}_{2}$ nanotubular films for improved VOC sensing Sensors and Actuators B:

Chemical 154 (2011) 251-256.

[7] N.H. Al-Hardan, M.J. Abdullah, A. Abdul Aziz, H. Ahmad, L.Y. Low, ZnO thin films for VOC sensing applications, Vacuum 85 (2010) 101-106.

[8] A.J. Ricco, R. M. Crooks, G.C. Osbourn, SAW Chemical Sensor Arrays: New Chemically Sensitive Interfaces Combined with Novel Cluster Analysis to Detect Volatile Organic Compounds and Mixtures, Account of Chemical Research 31 (1998) 289-296.

[9] C.K. Ho, E. R. Lindgren, K. S. Rawlinson, L. K. McGrath, J. L. Wright, Development of a Surface Acoustic Wave Sensor for In-Situ Monitoring of Volatile 
Organic Compounds, Sensors. 3 (2003) 236-247.

[10] N. Andreeva, T. Ishizaki, P. Barochc, N. Saitod, High sensitive detection of volatile organic compounds using superhydrophobic quartz crystal microbalance, Sensors and Actuators B: Chemical 164 (2012) 15-21.

[11] J. Homola, S.S. Yee, G. Gauglitz, Surface plasmon resonance sensors: review, Sensors and Actuators B: Chemical 54 (1999) 3-15.

[12] K. Kato, C.M. Dooling, K. Shinbo, T. H. Richardson, F. Kaneko, R. Tregonning, M.O. Vysotsky, C.A. Hunter, Gas sensing properties of porphyrin assemblies prepared using ultra-fast LB deposition, Colloids and Surfaces A: Physicochemical and Engineering Aspects 198-200 (2002) 811-816.

[13] S. Conoci, M. Palumbo, B. Pignataro, R. Rella, L. Valli, G. Vasapollo, Optical recognition of organic vapours through ultrathin calix[4]pyrrole films, Colloids and Surfaces A: Physicochemical and Engineering Aspects 198-200 (2002) 869-873.

[14] M.G. Manera, A. Colombelli, R. Rella, A. Caricato, P.D. Cozzoli, M. Martino, L. Vasanelli, $\mathrm{TiO}_{2}$ brookite nanostructured thin layer on magneto-optical surface plasmon resonance transductor for gas sensing applications, Journal of Applied Physics, 112, (2012) 053524.

[15] M.G. Manera, G. Leo, M.L. Curri, P.D. Cozzoli, R. Rella, P. Siciliano, A. 
Agostiano, L. Vasanelli, Investigation on alcohol vapours $/ \mathrm{TiO}_{2}$ nanocrystal thin films interaction by SPR technique for sensing application, Sensors and Actuators B: Chemical 100 (2004) 75-80.

[16] M.G. Manera, P.D. Cozzoli, G. Leo, M.L. Curri, A. Agostiano, L. Vasanelli, R. Rella, Thin films of $\mathrm{TiO}_{2}$ nanocrystals with controlled shape and surface coating for surface plasmon resonance alcohol vapour sensing, Sensors and Actuators B: Chemical 126 (2007) 562-572.

[17] M.G. Manera, P.D. Cozzoli, M.L. Curri, G. Leo, R. Rella, A. Agostiano, L. Vasanelli, $\mathrm{TiO}_{2}$ nanocrystal films for sensing applications based on surface plasmon resonance, Synthetic Metals 148 (2005) 25-29.

[18] E. Hutter, J.H. Fendler, Exploitation of Localized Surface Plasmon Resonance, Advanced Materials 16 (2004) 1685-1706.

[19] K.L. Kelly, E. Coronado, L.L. Zhao, G.C. Schatz, The Optical Properties of Metal Nanoparticles: The Influence of Size, Shape and Dielectric Environment, The Journal of Physical Chemistry B 107 (2003) 668-677.

[20] A.J. Haes, D.A. Stuart, S. Nie, R.P. Van Duyne, Using solution-phase nanoparticles, surfaceconfined nanoparticle arrays and single nanoparticles as biological sensing platforms, Journal of Fluorescence 14 (2004) 355-367. 
[21] S.-F. Cheng, L.-K. Chau, Colloidal Gold-Modified Optical Fiber for Chemical and Biochemical Sensing, Analytical Chemistry 75 (2003) 16-21.

[22] S. Kim, J.M. Jung, D.G. Choi, H.T. Jung, S.M. Yang, Patterned Arrays of Au Rings for Localized Surface Plasmon Resonance Langmuir 22 (2006) 109-7112.

[23] C.-S. Cheng, Y.-Q. Chen, C.-J. Lu, Organic vapour sensing using localized surface plasmon resonance spectrum of metallic nanoparticles self assemble monolayer, Talanta 73 (2007) 358-365.

[24] Y.-Q. Chen, C.-J. Lu, Surface modification on silver nanoparticles for enhancing vapor selectivity of localized surface plasmon resonance sensors, Sensors and Actuators B: Chemical 135 (2009) 492-498.

[25] J.M. Bingham, J.N. Anker, L.E. Kreno, R.P. Van Duyne, Gas Sensing with High-Resolution Localized Surface Plasmon Resonance Spectroscopy, Journal of the American Chemical Society. 132 (2010) 17358-17359.

[26] W.P. Hall, J.N. Anker, Y. Lin, J. Modica, M. Mrksich, R.P. Van Duyne, A Calcium-Modulated Plasmonic Switch, Journal of the American Chemical Society 130 (2008) 5836-5837.

[27] J.C. Hulteen, D.A. Treichel, M.T. Smith, M.L. Duval, T.R. Jensen, R.P. Van Duyne, Nanosphere lithography: Size-tunable silver nanoparticle and surface cluster 
arrays, The Journal of Physical Chemistry B 103 (1999) 3854-3863.

[28] J.C. Hulteen, R.P. Van Duyne, Nanosphere Lithography: A Materials General Fabrication Process for Periodic Particle Array Surfaces, Journal of Vacuum Science \& Technology A 13 (1995) 1553-1558.

[29] N. Geyer,; Z.P. Huang, B. Fuhrmann, S. Grimm, M. Reiche, T.K. Nguyen-Duc, J. de Boor, H.S. Leipner, P. Werner, U. Gosele, Sub-20 nm Si/Ge superlattice nanowires by metal-assisted etching, Nano Letters 9 (2009) 3106-3110.

[30] C.L. Haynes, A.D. McFarland, L.L. Zhao, G.C. Schatz; R.P. Van Duyne, L. Gunnarsson, J. Prikulis, B. Kasemo, M. Ka"1l, Nanoparticle Optics: The Importance of Radiative Dipole Coupling in Two-Dimensional Nanoparticle Arrays, The Journal of Physical Chemistry B 107 (2003) 7337-7342.

[31] H.D. Tong, J.V. Jansen, V.J. Gadgil, C.G. Bostan, E. Berenschot, C.J.M. van Rijn, Silicon Nitride Nanosieve Membrane, Nano Letters 4 (2004) 283-287.

[32] R.D. Piner, J. Zhu, F. Xu, S. Hong, C.A. Mirkin, "Dip-Pen" Nanolithography, Science 283 (1999) 661-663.

[33] K. Kosuge, S. Kubo, N. Kikukawa, M. Takemori, Effect of Pore Structure in Mesoporous Silicas on VOC Dynamic Adsorption/Desorption Performance, Langmuir 23 (2007) 3095-3102. 
[34] B.C. Galarreta, E. Harte, N. Marquestaut, P.R. Norton, F. Lagugne-Labarthet, Plasmonic properties of Fischer's patterns: polarization effects, Physical Chemistry Chemical Physics 12 (2010) 6810-6816.

[35] S. Kim, N. Cheng, J.R. Jeong, S.G. Jang, S.M. Yang, W.T. Huck, Localized surface plasmon resonance (LSPR) sensitivity of Au nanodot patterns to probe solvation effects in polyelectrolyte brushes, Chemical Communications 31 (2008) 3666-3668.

[36] T. Sasahara, A. Kido, H. Ishihara, T. Sunayama, M. Egashira, Highly sensitive detection of volatile organic compounds by an adsorption/combustion-type sensor based on mesoporous silica, Sensors and Actuators B: Chemical 108 (2005) 478-483.

[37] T.R. Jensen, M.D. Malinsky, C.L. Haynes, R.P. Van Duyne, Nanosphere lithography: tunable localized surface plasmon resonance spectra of silver nanoparticles, The Journal of Physical Chemistry B 104 (2000) 10549-10556.

[38] L.E. Kreno, J.H. Hupp, R.P. Van Duyne, Model of Vapor-Induced Resistivity Changes in Gold-Thiolate Monolayer-Protected Nanoparticle Sensor Films, Analytical Chemistry 79 (2007) 4977-4986.

[39] A.A. Isirikyan, A.V. Kiselev, The absolute adsorption isotherms of vapors of nitrogen, benzene and n-hexane, and heats of adsorption of benzene and n-hexane on graphitized carbon blacks, I. Graphitized Thermal Blacks, The Journal of Physical 
Chemistry B 65 (1961) 601-607.

[40] A. Sayari, E. Crusson, S. Kaliaguine, J.R. Brown, External surface areas of H-ZSM 5 zeolites, Langmuir 7 (1991) 314-317.

[41] K.S.W. Sing, D.H. Everett, R.A.W. Haul, L. Moscou, R.A. Pierotti, J. Rouquérol, T. Siemieniewska, Reporting Physisorption Data for gas/Solid Systems with Special Reference to the Determination of Surface Area and Porosity, Pure and Applied Chemistry 57 (1985) 603-619. 


\section{Biographies}

Akira Monkawa received his Ph.D. from the University of Tokyo in 2004. Currently, he is a Senior Researcher in the Tokyo Metropolitan Industrial Technology Research Institute. His research interest is the optical sensor based on the local surface plasmon resonance.

Tomoe Nakagawa received her M. S. degree from Shizuoka University in 2009. Currently, she is a Chief Researcher in Tokyo Metropolitan Industrial Technology Research Institute. Her research interest is the organic chemistry.

Hirokazu Sugimori received his Ph.D. from the University of Tokyo in 2007. Currently, he is a Chief Researcher in Tokyo Metropolitan Industrial Technology Research Institute. His research interest is environmental engineering and geochemistry.

Elito Kazawa received her B. S. degree from The University of ElectroCommunications in 1986. Currently, he is a Senior Researcher in Tokyo Metropolitan Industrial Technology Research Institute. His research interest is fine pattern lithography. 
Assistant Prof. Dr. Kohei Shibamoto received his PhD degree from Department of Advanced Materials Science, Graduate School of Frontier Science, The University of Tokyo in 2004. He has 15 years of research experiences in the area of laser spectrometry and optical sensor. In particular, he has been focusing on fabrication of two-dimensional close packed array of gold nano-particle. Currently, he is an assistant professor of Tokyo Metropolitan University, Department of Chemistry.

Associate Prof. Dr. Takashi Takei received his $\mathrm{PhD}$ and MEng degree from Tokyo Metropolitan University. He was employed as a researcher in the Central Research Laboratory of Nissan Chemical Industry Co. Ltd from 1983 to 1986. In 1986 he worked as a research associate at the Dep. Industrial Chemistry, Tokyo Metropolitan University, His research interest is the effects of oxide supports on gold catalysis. Currently, he is an associate professor of the Dep. Industrial Chemistry in the Graduate School of Urban Environmental Sciences, Tokyo Metropolitan University.

Prof. Dr. Masatake Haruta received his $\mathrm{PhD}$ degree from the Graduate School of Kyoto University, Faculty of Engineering, Department of Industrial Chemistry in 1976. He has 40 years of research experiences in the area of chemical sensors and 
heterogeneous catalysts. In particular, he has been focusing his efforts on the creation of novel catalysis by gold nanoparticles, which can lead to green chemistry. Currently, he is a full professor of Tokyo Metropolitan University, Faculty of Urban Environmental Sciences, Department of Applied Chemistry. He is also a visiting professor at Dalian Institute of Chemical Physics. 
Table

Table 1. The slope of calibration curves (10-100ppm and 100-1000ppm), LOD and vapor pressure of tested VOCs

\begin{tabular}{lcccc} 
& Slope $(10-100 \mathrm{ppm})$ & Slope $(100-1000 \mathrm{ppm})$ & LOD & $\mathrm{Pv}$ \\
\hline Benzene & 2.20 & 1.12 & 1.93 & 100.8 \\
Toluene & 10.93 & 2.06 & 0.40 & 28.5 \\
o-xylene & 13.50 & 9.60 & 0.32 & 6.6 \\
p-xylene & 16.20 & 7.64 & 0.26 & 8.8 \\
m-xylene & 11.05 & 7.38 & 0.38 & 8.3 \\
Ethyl benzene & 9.62 & 6.83 & 0.44 & 9.5 \\
Chlorobenzene & 6.17 & 4.46 & 0.69 & 11.8 \\
Methanol & 2.51 & 0.75 & 1.69 & 127.1 \\
Ethanol & 6.26 & 1.97 & 0.68 & 59.0 \\
1-propanol & 15.58 & 2.26 & 0.27 & 20.5 \\
2-propanol & 8.11 & 1.64 & 0.52 & 42.7 \\
1-butanol & 25.28 & 4.95 & 0.17 & 6.2 \\
cis-DCE & 0.43 & 0.38 & 9.92 & 200.0 \\
$\mathrm{TCE}^{-}$ & 0.85 & 0.85 & 4.99 & 69.0 \\
$\mathrm{PCE}^{-14}$ & 2.92 & 1.67 & 1.46 & 18.5 \\
$\mathrm{CH}_{2} \mathrm{Cl}{ }_{2}$ & 0.22 & 0.21 & 18.92 & 435.9 \\
$\mathrm{CHCl}_{3}$ & 0.61 & 0.60 & 6.97 & 194.2 \\
$\mathrm{CCl}_{4}$ & 0.40 & 0.47 & 10.70 & 115.3 \\
\hline
\end{tabular}


Fig. captions

Fig. 1. The schematic diagram of VOC sensing system. a) clean air unit, b) standard gas-generation system, c) bubbler, d) mixing chamber, e) mass-flow controller, f) sensor.

Fig. 2. The LSPR spectra of five different Au dot patterns calculated by OptiFDTD.

Fig. 3. The SEM images of Au dot patterns fabricated by the EBL method. a) D100P200 and b) D400P800.

Fig. 4. The LSPR spectra of five different Au dot patterns fabricated by the EBL method.

Fig. 5. The change of the LSPR spectrum after porous silica coating.

Fig. 6. The LSPR spectrum changes when exposed to different relative saturation concentrations $(0-100 \%)$ of toluene. 
Fig. 7. The dynamic response of the LSPR sensor in the range of toluene gas

concentration from $20000 \mathrm{ppm}$ to $10 \mathrm{ppm}$.

Fig. 8. a) The toluene adsorption isotherms of porous silica powder estimated by the

LSPR sensor. b) The toluene adsorption isotherms of porous silica powder obtained

from static adsorption experiment.

Fig. 9. The dynamic response of the sensing element in the presence of toluene of $1-50$

ppm.

Fig. 10. The calibration curves obtained from the dynamic response at every $50 \mathrm{~s}$ after the introduction of toluene gas.

Fig. 11. The calibration curves of (a) aromatic, (b) alcohol, (c) chloroethylene, (d) chloromethane VOCs in the range of 10-1000 ppm.

Fig. 12. Adsorption isotherms of (a) aromatic, (b) alcohol, (c) chloroethylene, (d) chloromethane VOCs. 
Fig. 13. The correlation between the amount of shift and refractive index of VOCs.

Fig. 14. The interaction between sensitivity of the sensor (count/ppm) and reciprocal of VOCs saturated vapor pressure $(1 / \mathrm{Pv})$. 
Figure 1.

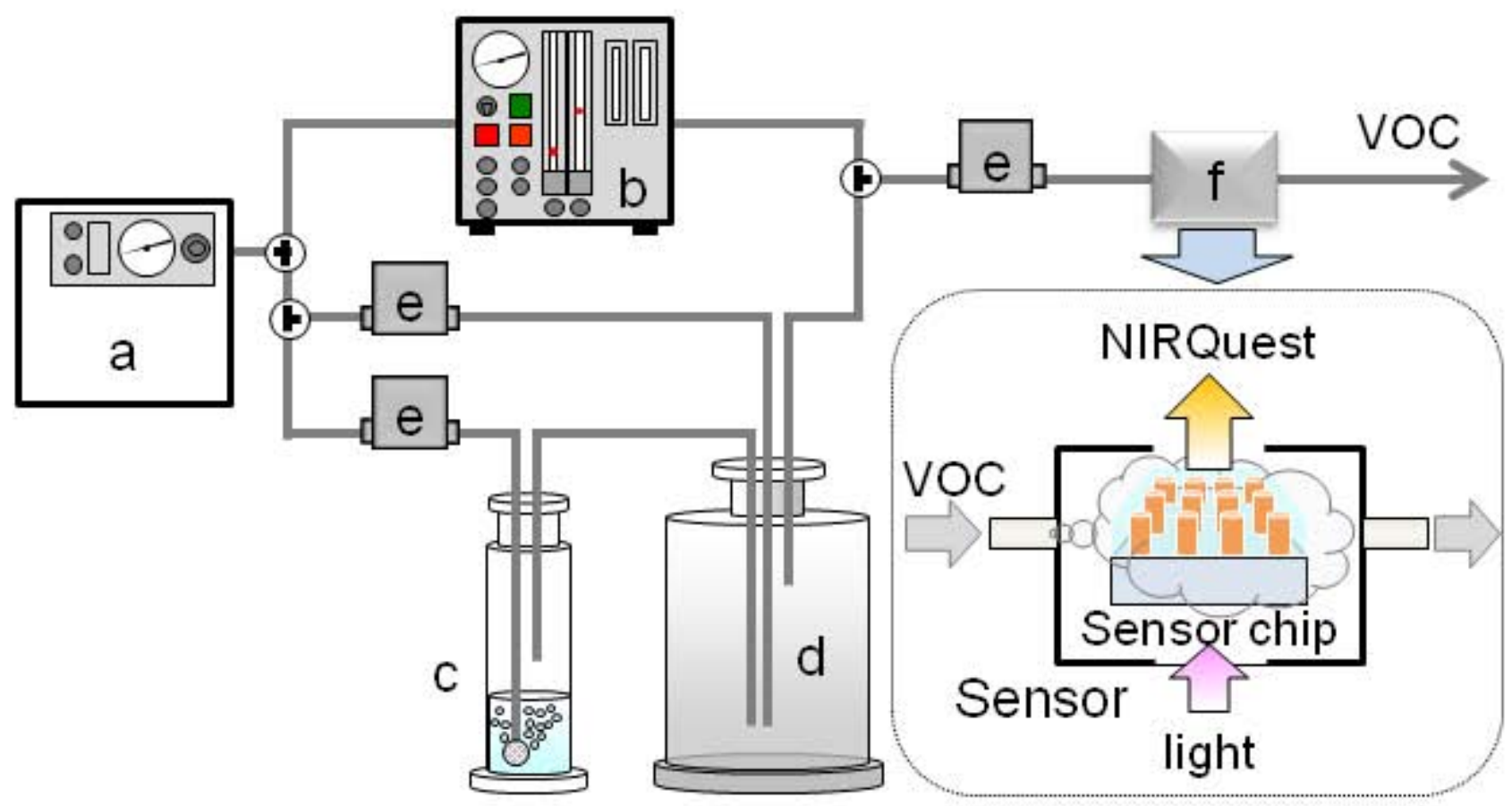


Figure 2.

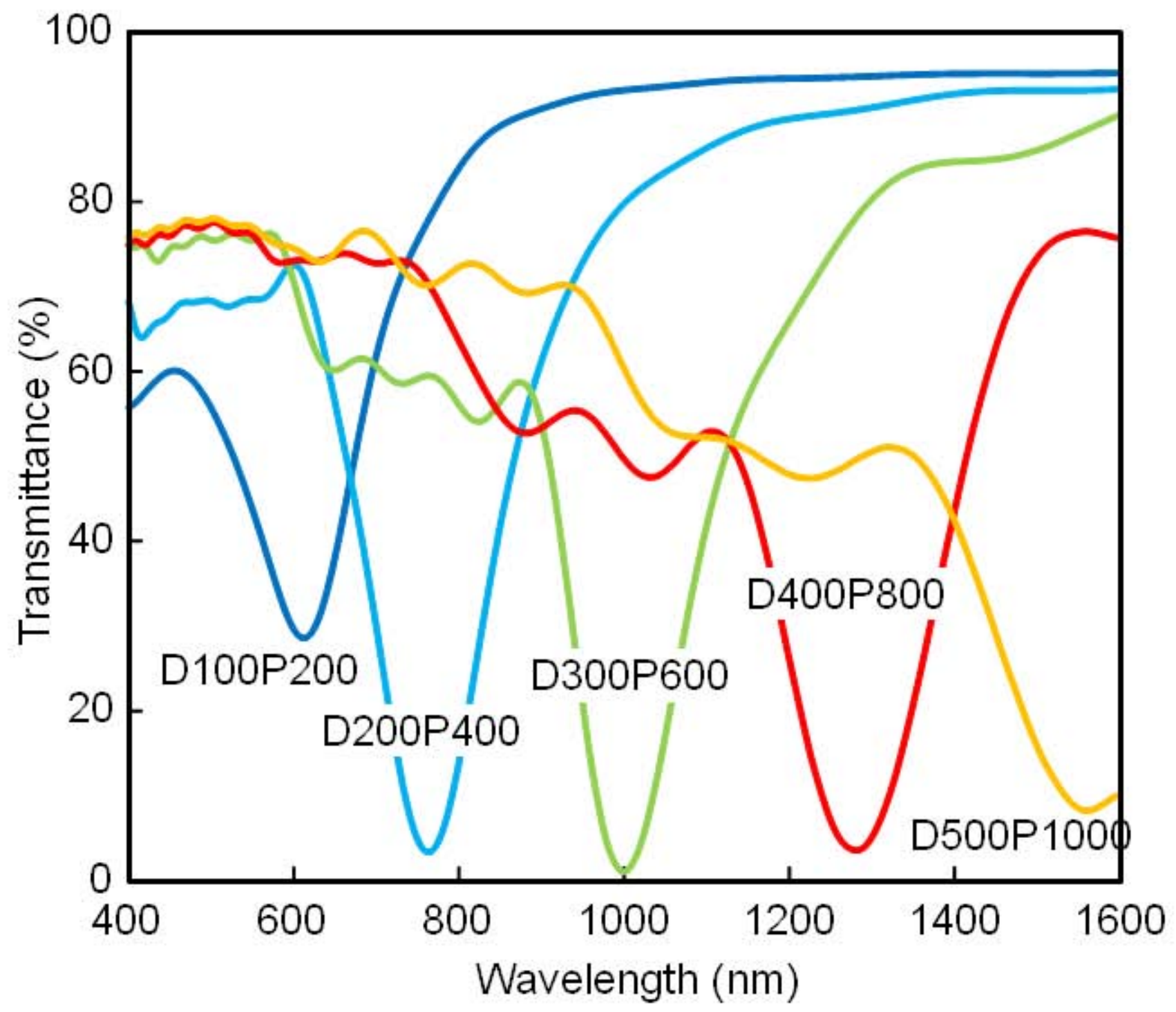


Figure 3.
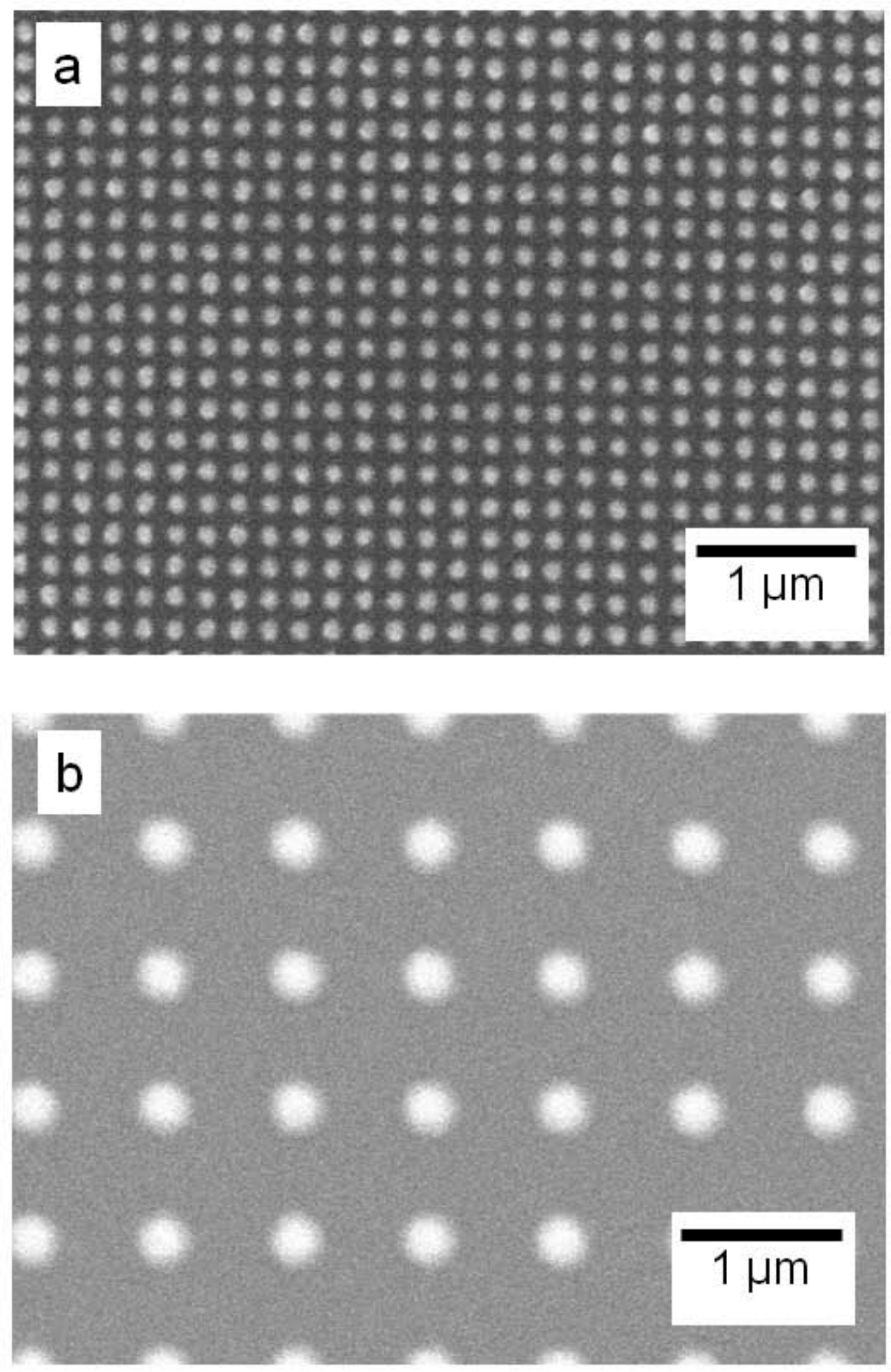
Figure 4.

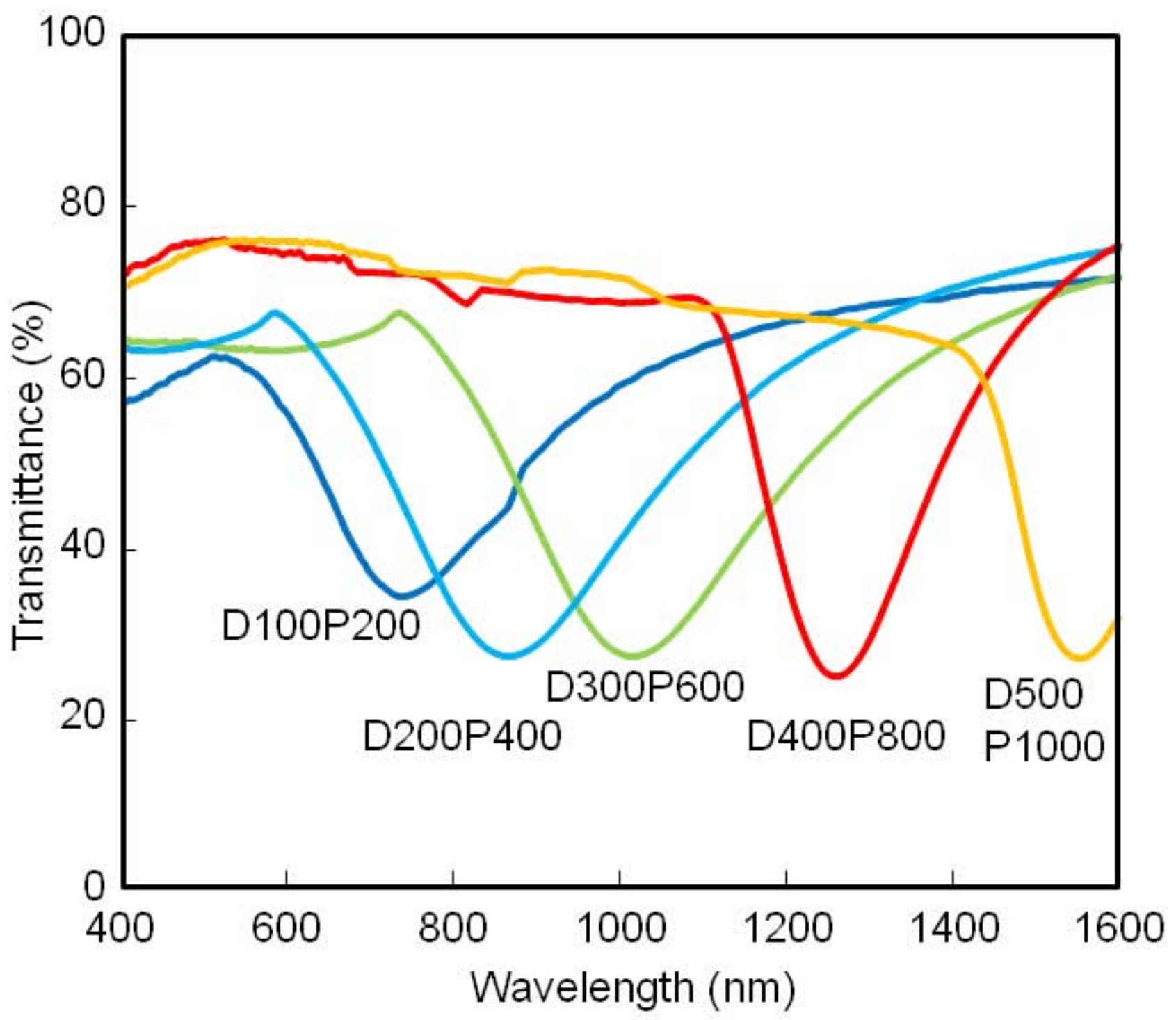


Figure 5.

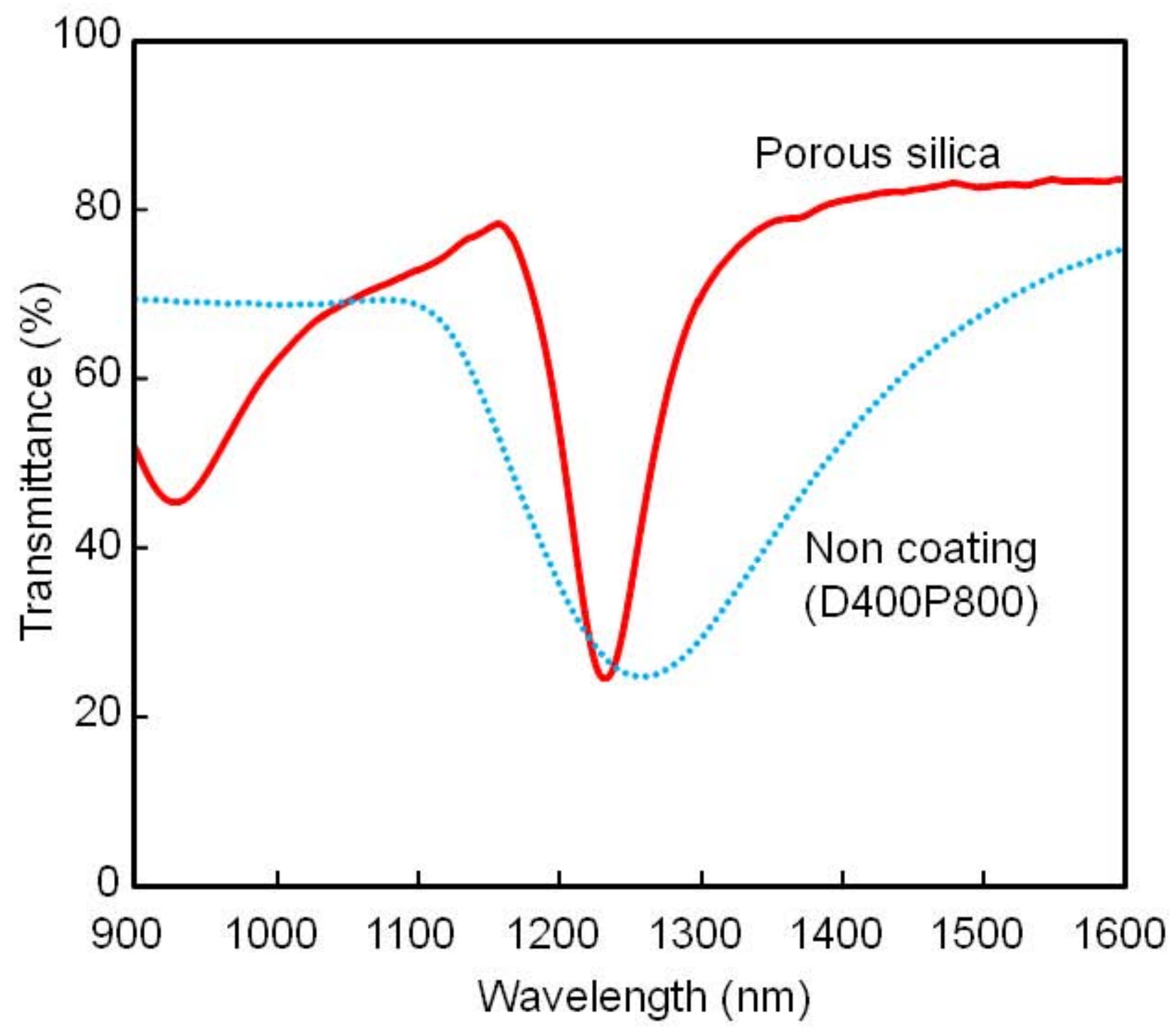


Figure 6.

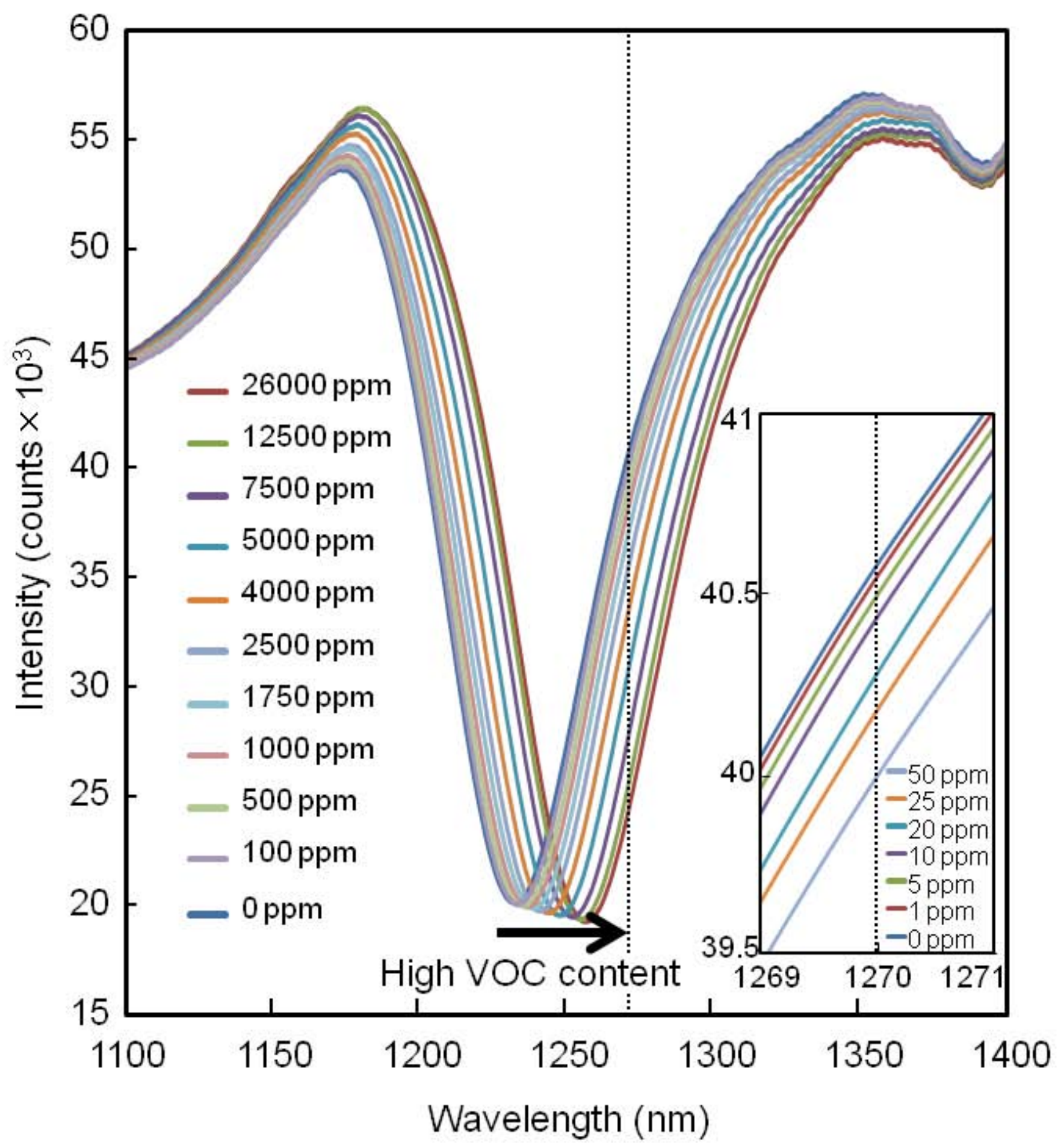


Figure 7.
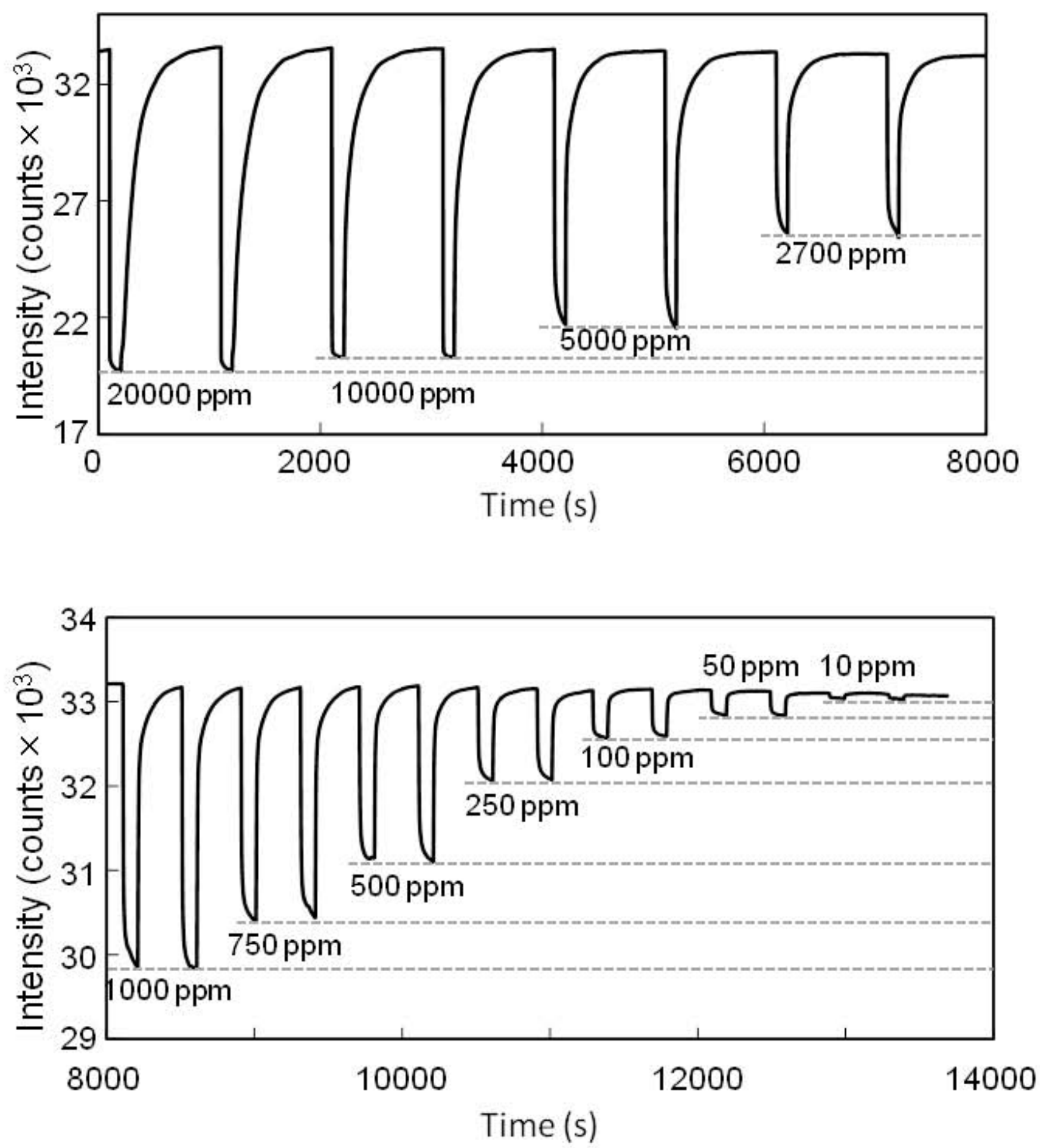
Figure 8.
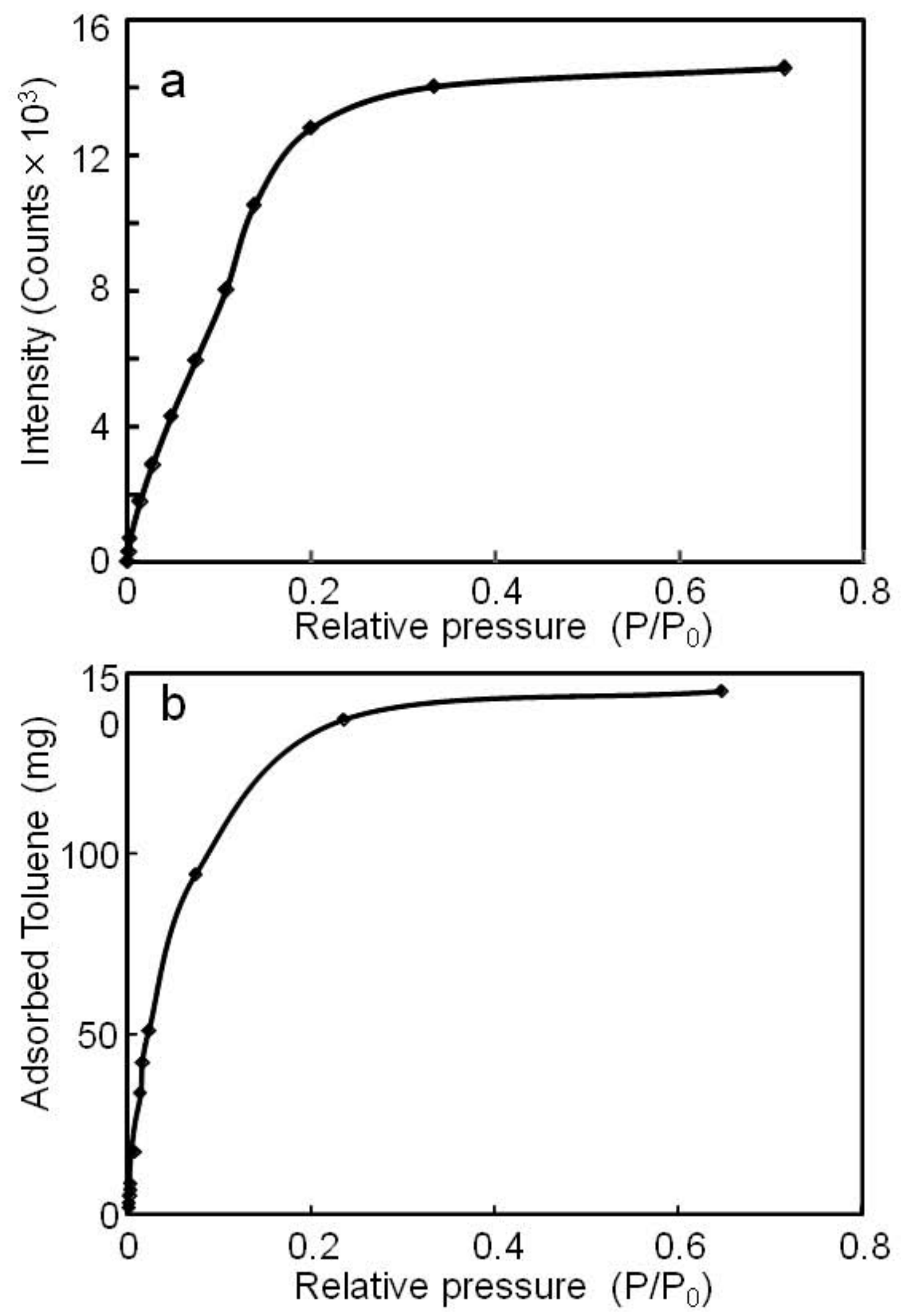
Figure 9.

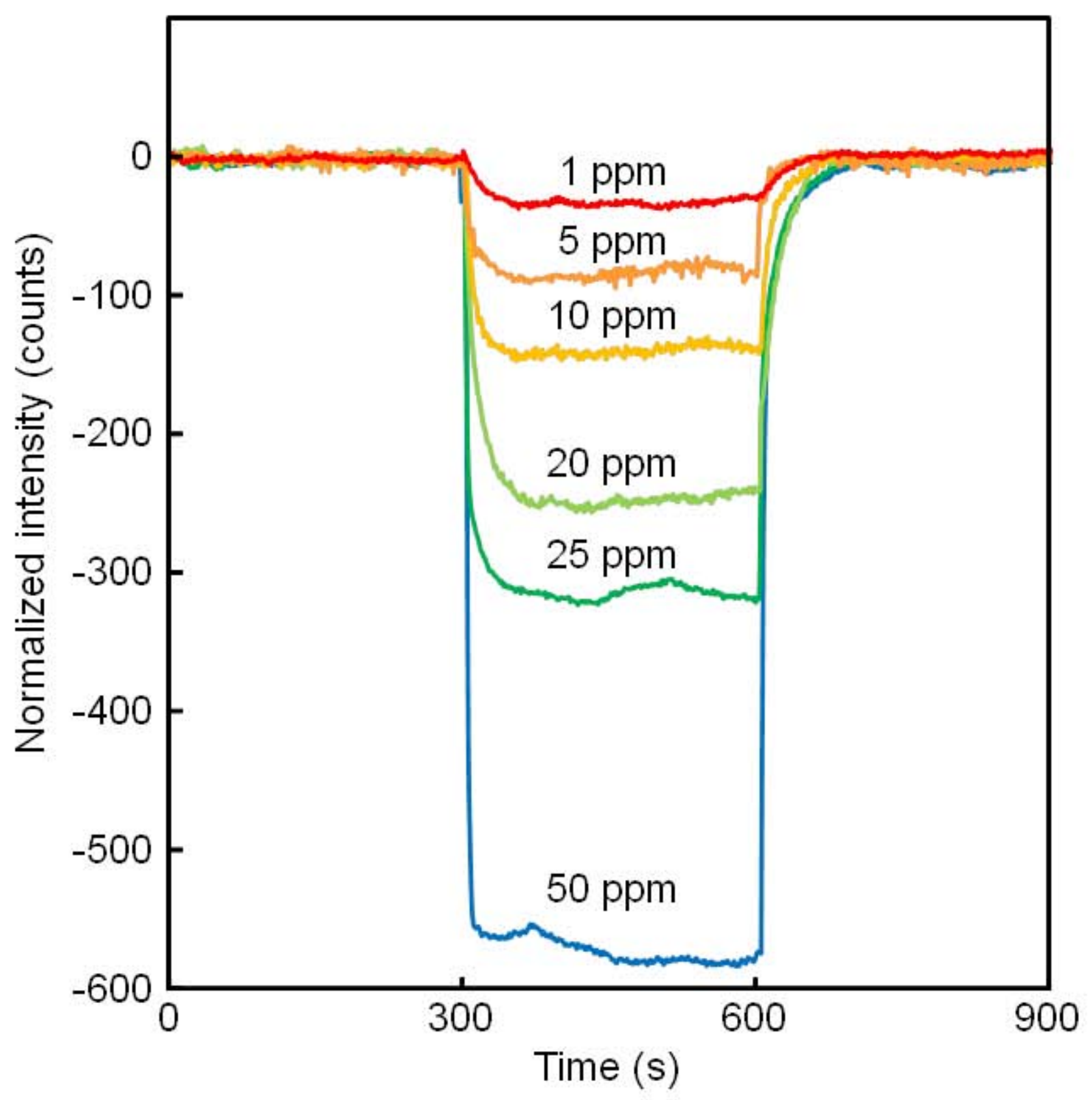


Figure 10.

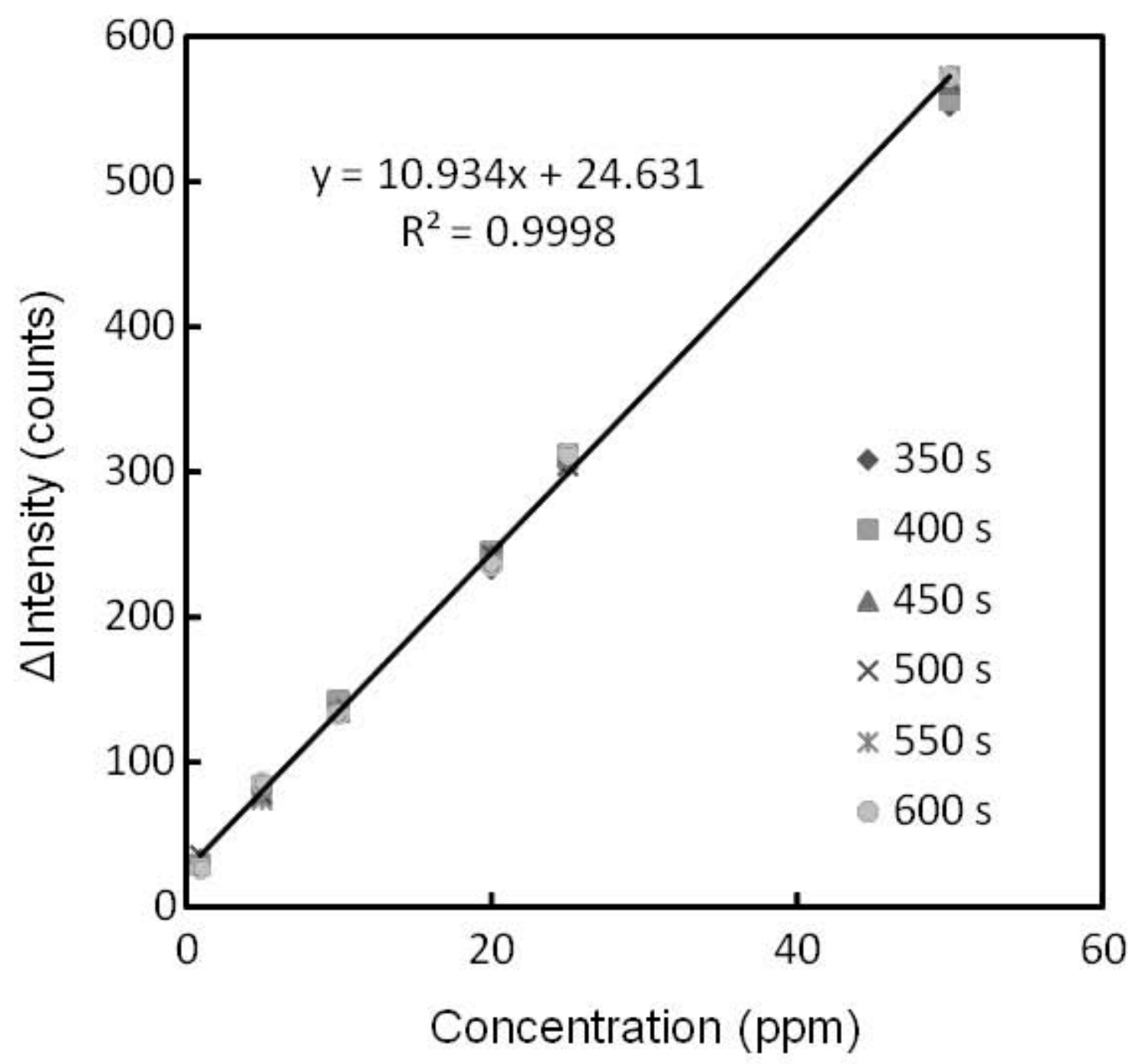


Figure 11.
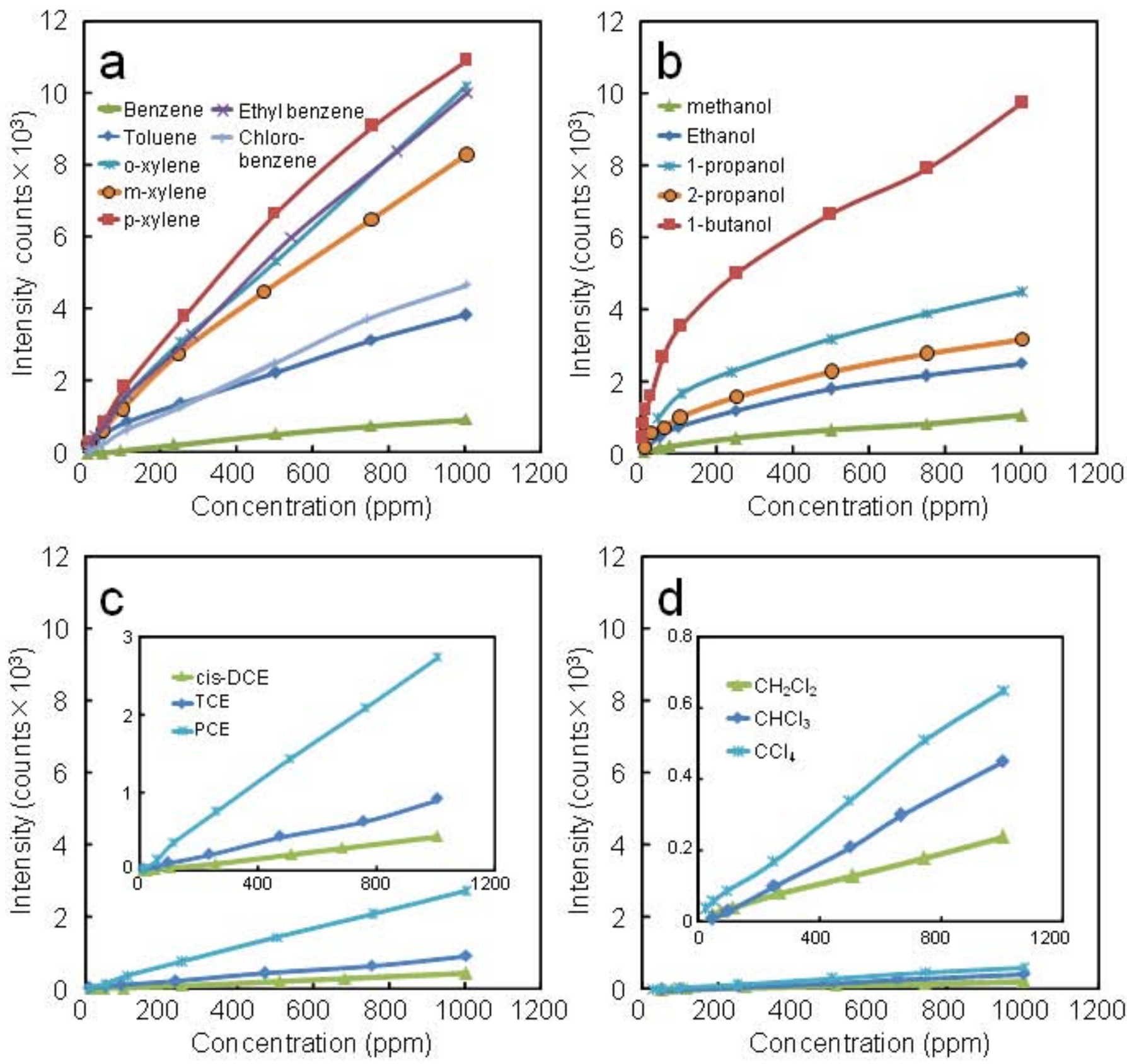
Figure 12.
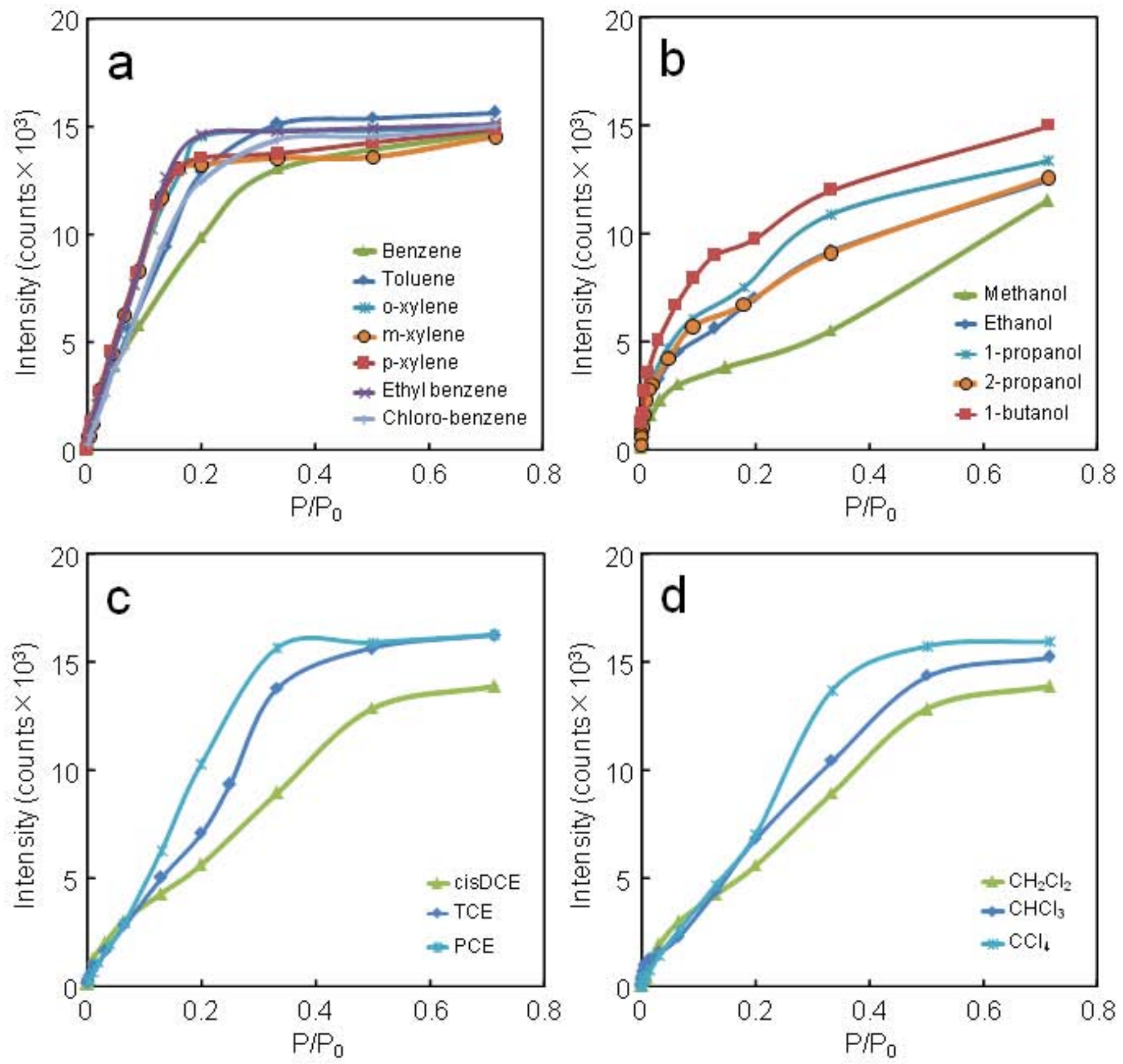
Figure 13.

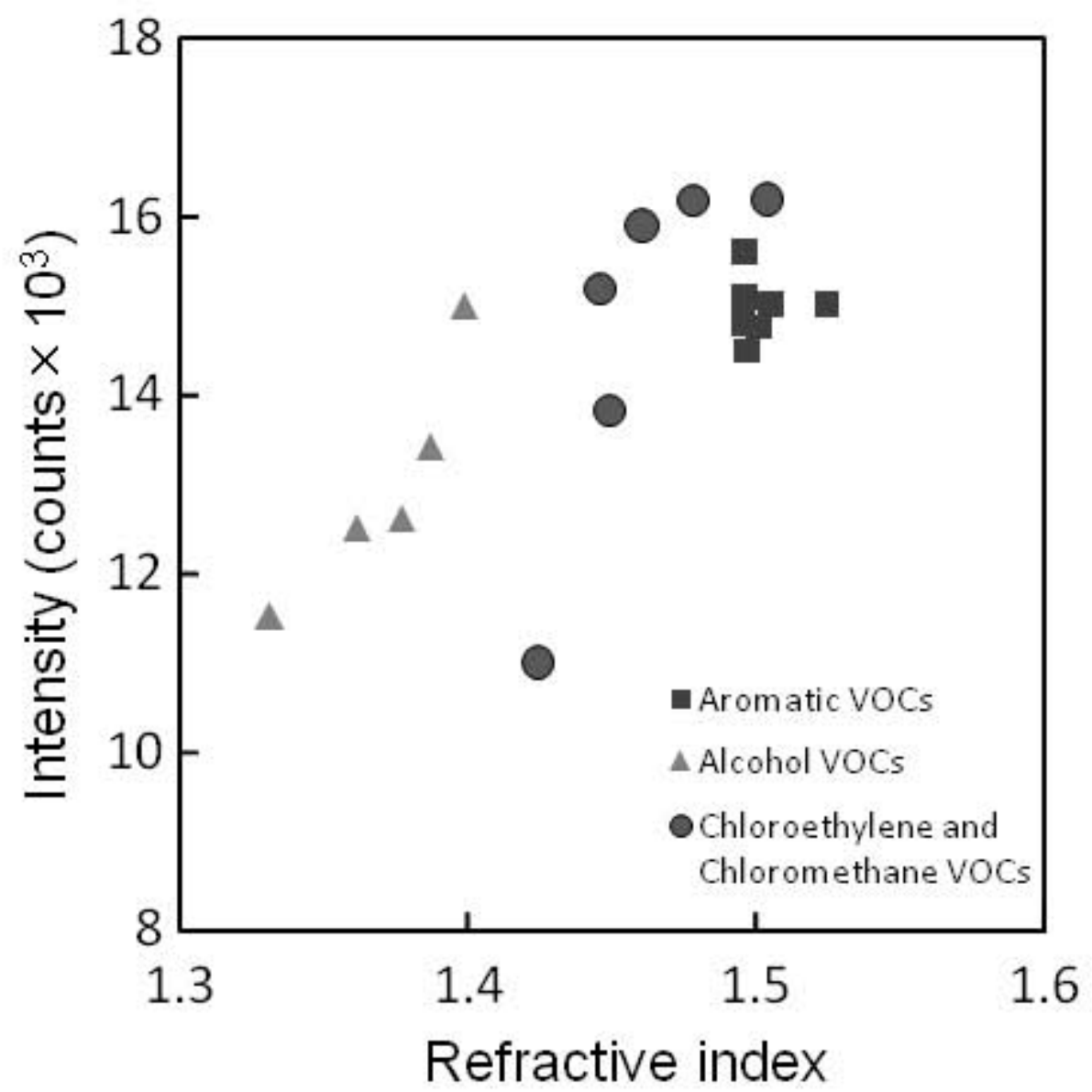


Figure 14.

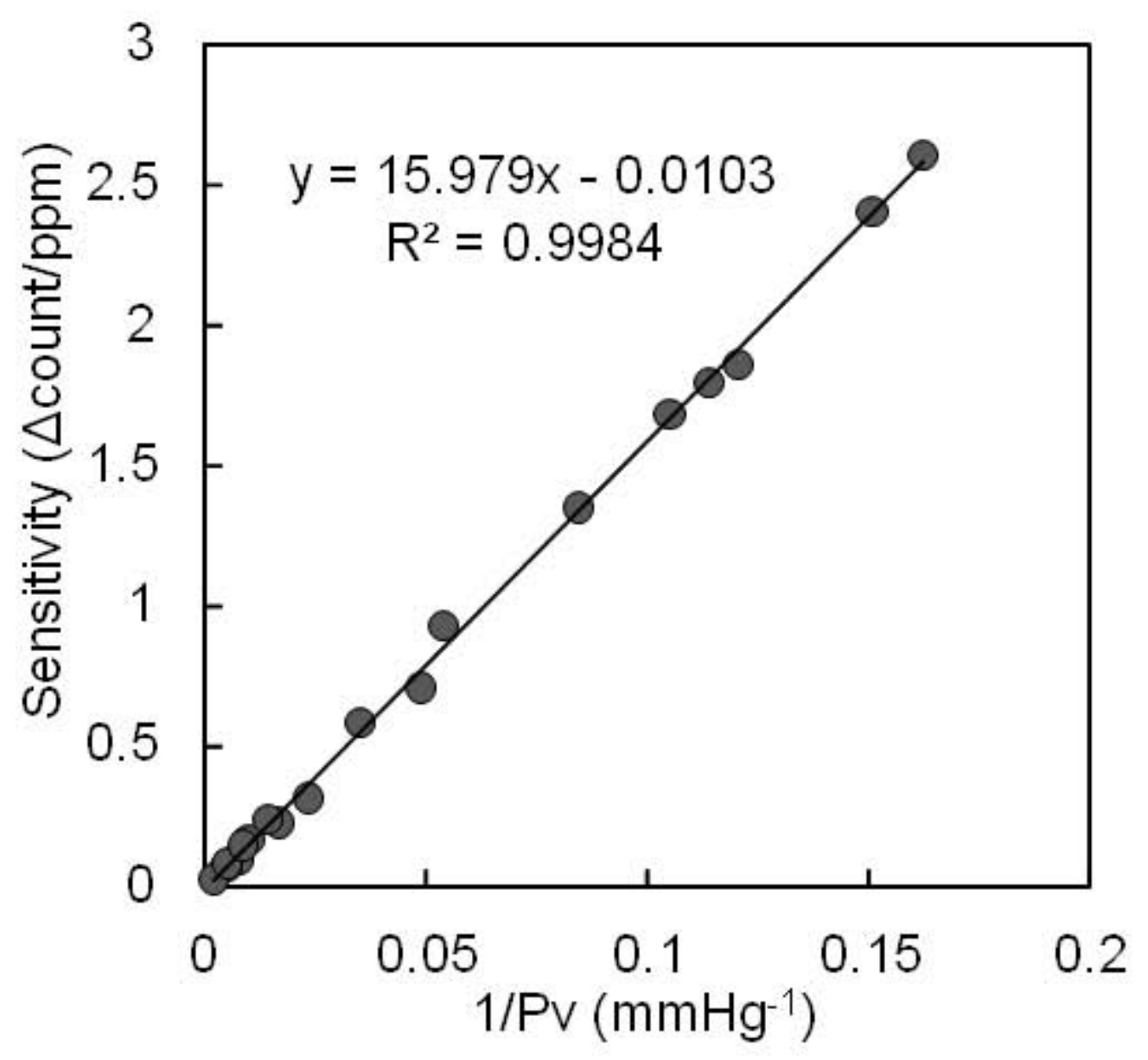

